\title{
Resultados de la cirugía torácica mínimamente invasiva (vídeo-asistida) en el tratamiento de la Miastenia Gravis*
}

\author{
Drs. JAIME JANS B. ${ }^{1}$, ROBERTO GONZÁLEZ L. ${ }^{2}$
}

Servicio de Cirugía, Hospital Base Osorno. Campo Clínico Osorno. Facultad de Medicina, Universidad Austral de Chile. Osorno.

2 Servicio de Cirugía, Equipo de Cirugía Cardiotorácica. Hospital Clínico Regional "Dr. Guillermo Grant Benavente”, Concepción. Facultad de Medicina, Universidad de Concepción. Concepción. Chile.

\section{Abstract \\ Results of minimally invasive thoracic surgery (video-assisted) in the treatment of Myasthenia Gravis}

Introduction: Myasthenia Gravis (MG) is the most common primary disorder of neuromuscular transmission. Treatment is multidisciplinary medical and surgical. Thymectomy is now part of standard treatment with clinical improvement in approximately $85 \%$ of cases. The best approach has not yet been defined. Objective: To evaluate the results of thymectomy for minimally invasive surgery (video-assisted) in patients with MG, with or without thymoma, analyzing the results in the literature to date. Material and Methods: We conducted a systematic review of literature in the PubMed database. Results: We found 66 publications related to the search, 20 studies were selected for review. We included those that evaluated the results of thymectomy in MG patients with or without thymoma. Discussion: Minimally invasive surgery (video-assisted) is a safe way, with low morbidity, shorter hospitalization, better cosmetic result and complete remission rates or improvement at least equivalent to conventional surgical approaches. These results should be evaluated with caution because there isn't enough evidence to ensure that minimally invasive surgery is the best approach.

Key words: Thoracic surgical procedures, videothoracoscopy, surgical procedures, minimally invasive, myasthenia gravis, surgical treatment.

\section{Resumen}

Introducción: La Miastenia Gravis (MG) es el desorden primario más común de la transmisión neuromuscular. Su tratamiento es multidisciplinario, médico y quirúrgico. La timectomía es en la actualidad parte del estándar de tratamiento con mejorías clínicas en aproximadamente el $85 \%$ de los casos. El mejor abordaje aún no se ha definido. Objetivo: Evaluar los resultados de la timectomía por cirugía mínimamente invasiva (video-asistida) en pacientes con MG, con o sin timoma, analizando los resultados en la literatura disponible hasta la actualidad. Material y Métodos: Se realizó una revisión sistemática de la literatura en la base de

*Recibido el 27 de agosto de 2012 y aceptado para publicación el 28 de septiembre de 2012.

No existen conflictos de interés ni apoyo financiero.

Correspondencia: Dr. Jaime Jans B.

Av. Guillermo Bühler 1712, Osorno, Chile.

jaimejans@gmail.com 
datos PubMed. Resultados: Se encontró 66 publicaciones relacionadas con la búsqueda, de los cuales se seleccionó 20 estudios para revisión. Se incluyó aquellos en los que se evaluó los resultados de la timectomía en pacientes con MG, con o sin timoma. Discusión: La cirugía mínimamente invasiva (video-asistida) es una vía segura, con tasas de morbilidad bajas, menor tiempo de hospitalización, buen resultado estético y tasas de remisión completa o de mejoría al parecer equivalentes a las vías de abordaje clásicas. Estos resultados deben evaluarse con cautela ya que no existe suficiente evidencia para asegurar que la cirugía mínimamente invasiva es el mejor abordaje.

Palabras clave: Procedimientos quirúrgicos torácicos, videotoracoscopia, cirugía mínimamente invasiva, miastenia Gravis, tratamiento quirúrgico.

\section{Introducción}

La Miastenia Gravis (MG) es una enfermedad autoinmune que se caracteriza por la presencia de debilidad muscular fluctuante y fatiga de distintos grupos musculares. Los responsables de la falla en la transmisión sináptica que producen las manifestaciones clínicas son los anticuerpos dirigidos contra los receptores nicotínicos de acetilcolina de la membrana postsináptica en la unión neuromuscular. La prevalencia es aproximadamente de 5 casos $/ 100.000$ personas. Afecta a individuos de todas las edades, con una predilección por mujeres entre 20 y 40 años y varones entre 60 y 70 años ${ }^{1}$. Clínicamente se caracteriza por debilidad y fatiga muscular que afecta a distintos grupos de músculos. La debilidad muscular empeora con la actividad física y mejora con el reposo. Los músculos oculares, faciales y bulbares (músculos que controlan el habla, la deglución y la masticación) son los más afectados por la enferme$\mathrm{dad}^{2}$. Según el momento de presentación se divide en Miastenia infantil (neonatal, congénita y juvenil) y Miastenia del Adulto. Esta última se estadifica según la clasificación de Osserman y Genkins modificada $^{3}$ en 4 estadios clínicos. Otras clasificaciones, como el sistema de clasificación de Drachman ${ }^{4}$ y la Clasificación Clínica de la Fundación de Miastenia Gravis de América ${ }^{5}$, han sido utilizadas con el objeto de evaluar de forma más objetiva y consensuada los resultados del tratamiento médico-quirúrgico.

El tratamiento es multidisciplinario (neurólogos, médicos internistas, oftalmólogos y cirujanos). El tratamiento médico consiste en el uso de anticolinesterásicos, como la piridostigmina, para el tratamiento de los síntomas y de inmunosupresores, como los corticoides, para tratar la autoinmunidad subyacente.

El tratamiento quirúrgico fue comunicado por primera vez en 1912 por Sauerbruch ${ }^{6}$. En 1939, Blalock ${ }^{7}$, realizó una timectomía transesternal en una paciente con un quiste tímico y MG, que resultó en la mejoría sintomática. Posteriormente, publicó resultados favorables de la timectomía en pacientes con MG sin timoma ${ }^{8}$. Desde esos años, la experiencia y sus resultados han hecho de la timectomía parte fundamental del tratamiento de la MG, a pesar de la ausencia de estudios prospectivos randomizados que comparen la cirugía con el tratamiento médico. Lo más cercano a dicha evidencia es la comunicación de Buckingham en 1976 en la Clínica Mayo9, donde realizaron el análisis retrospectivo de pacientes tratados con timectomía por MG, pareándolos con una cohorte histórica de pacientes con características similares tratados farmacológicamente. $\mathrm{La}$ conclusión fue que los pacientes tratados en forma médica tenían un $8 \%$ de remisión (definida como la ausencia de medicamentos y de síntomas) y un $43 \%$ de mortalidad versus un $34 \%$ de remisión y $14 \%$ de mortalidad en la cohorte quirúrgica.

En la actualidad la timectomía como terapia adyuvante de la MG es considerada el estándar de tratamiento, sin embargo, la vía de abordaje sigue siendo controversial. En los últimos años se ha extendido el uso de técnicas mínimamente invasivas como la videotoracoscopia o cirugía torácica video asistida (VATS).

El objetivo de esta revisión es evaluar los resultados de la timectomía por técnicas mínimamente invasivas (video-asistida) en pacientes con $\mathrm{MG}$, con o sin timoma, analizando los resultados en la literatura disponible hasta la fecha.

\section{Material y Método}

Se realizó una revisión sistemática de la literatura en la base de datos PubMed, período 1990-2011, utilizando los términos Mesh: "Surgical Procedures, Minimally Invasive" and "Thoracic Surgery, VideoAssisted" and "Myasthenia Gravis". Los límites de búsqueda fueron estudios en humanos y en idioma inglés.

Se seleccionó estudios en base a los siguientes criterios de inclusión:

1) Publicado en revistas con disponibilidad de texto completo.

2) Que incluyeran el análisis de resultados inmediatos y/o clínicos de la timectomía con técnicas mínimamente invasivas.

3) Se complementó con artículos adicionales en base a las referencias de los artículos antes seleccionados. 
Se entrega un comentario de los estudios y se resumen los resultados quirúrgicos y clínicos en tablas comparativas.

\section{Resultados}

Con los criterios de búsqueda descritos se encontró 66 artículos. De estos, 20 se seleccionaron para el análisis. Trece corresponden a estudios retrospectivos y 7 a prospectivos. Ocho estudios corresponden a series de casos y 12 a estudios comparativos entre 2 o más abordajes quirúrgicos. Las características y resultados globales se resumen en las Tablas 1 y 2.

Yim et al, (1995) ${ }^{10}$ compararon retrospectivamente los resultados de la timectomía por VATS

Tabla 1. Tipos de estudios, abordajes y resultados

\begin{tabular}{|c|c|c|}
\hline Autor / Estudio & Abordajes (pacientes) & Resultados \\
\hline $\begin{array}{l}\text { Yim et al., } 1995^{10} \\
\text { Cohorte Retrospectiva } \\
1993 \text { - } 1994 \text { (nivel 2b)* } \\
\text { Con y sin timoma } \\
\text { Osserman I-IV }\end{array}$ & $\begin{array}{ll}\text { VATS } & (\mathrm{n}=8) \\
\text { TE } & (\mathrm{n}=8)\end{array}$ & $\begin{array}{l}\text { Tiempo op: } 70,8 \pm 11,6 \text { vs } 107,8 \pm 22,2 \text { min } \\
\text { ( } p<0,05 \text { ) } \\
\text { Hospitalización: } 4,3 \pm 0,9 \text { vs } 9,6 \pm 1,4 \text { días } \\
(p<0,05) \\
\text { Morbilidad: } 12 \%\end{array}$ \\
\hline $\begin{array}{l}\text { Mineo et al., } 2000^{11} \\
\text { Cohorte prospectiva } \\
1993 \text { - } 1997 \text { (nivel } 1 b)^{*} \\
\text { Con y sin timoma } \\
\text { Osserman I-IV }\end{array}$ & VATS $\quad(n=31)$ & $\begin{array}{l}\text { Tiempo op: } 148 \pm 46 \min \\
\text { Hospitalización: } 5,2 \pm 2,8 \text { días } \\
\text { Morbilidad: } 3,2 \% \\
\text { Conversión: } 3,2 \%\end{array}$ \\
\hline $\begin{array}{l}\text { Savcenko et al., } 2002^{12} \\
\text { Cohorte retrospectiva } \\
1992 \text { - } 2002 \text { (nivel } 2 b)^{*} \\
\text { No especifica timoma } \\
\text { MGFA I-V }\end{array}$ & VATS $\quad(n=36)$ & $\begin{array}{l}\text { Tiempo op: NE } \\
\text { Hospitalización: } 1,64 \pm 1,86 \text { días } \\
\text { Morbilidad: } 5,5 \% \\
\text { Conversión: } 2,6 \%\end{array}$ \\
\hline $\begin{array}{l}\text { Wright et al., } 2002^{13} \\
\text { Cohorte prospectiva } \\
1997-2001 \text { (nivel 1b)* } \\
\text { Sin timoma } \\
\text { Osserman NE }\end{array}$ & VATS $\quad(n=26)$ & $\begin{array}{l}\text { Tiempo op: } 1 \text { a } 3 \text { h } \\
\text { Hospitalización: mediana } 4 \text { días (rango 2-6) } \\
\text { Morbilidad: } 15,4 \% \\
\text { Conversión: } 3,84 \%\end{array}$ \\
\hline $\begin{array}{l}\text { Ruckert el al., } 2003^{14} \\
\text { Cohorte Retrospectiva } \\
1983 \text { - } 2000 \text { (nivel } 2 b)^{*} \\
\text { Sin timoma } \\
\text { Osserman I-III }\end{array}$ & $\begin{array}{ll}\text { VATS } & (\mathrm{n}=20) \\
\text { TE } & (\mathrm{n}=20) \\
\text { TAL } & (\mathrm{n}=20) \\
\text { (pareada } & \text { por edad, género y Osserman) }\end{array}$ & $\begin{array}{l}\text { Tiempo op: } 197 \text { vs } 113 \text { vs } 82 \min (\mathrm{p}<0,001) \\
\text { Hospitalización: NE } \\
\text { Morbilidad: } 5 \% \text { vs } 25 \% \text { vs } 15 \%(\mathrm{p}<0,05)\end{array}$ \\
\hline $\begin{array}{l}\text { Mantegazza et al., } 2003^{15} \\
\text { Comparativo prospectivo } \\
\text { Período NE (nivel 1b)* } \\
\text { Sin timoma } \\
\text { MGFA I-IV }\end{array}$ & $\begin{array}{l}\text { BVATS + cervicotomía }(\mathrm{n}=159) \\
\text { TE } \quad(\mathrm{n}=47)\end{array}$ & $\begin{array}{l}\text { Sólo BVATS: } \\
\text { Tiempo op: } 84,3 \pm 25,1 \mathrm{~min} \\
\text { Hospitalización: } 5 \pm 2 \text { días } \\
\text { Morbilidad: } 0,62 \%\end{array}$ \\
\hline $\begin{array}{l}\text { Hsu et al., } 2004^{16} \\
\text { Comparativo prospectivo } \\
2001-2003 \text { (nivel 1b)* } \\
\text { Con y sin timoma } \\
\text { Osserman I-III }\end{array}$ & $\begin{array}{l}\text { BVATS }+ \text { subxifoideo }(\mathrm{n}=15) \\
\text { VATS } \quad(\mathrm{n}=12)\end{array}$ & $\begin{array}{l}\text { Tiempo op: } 151,3 \text { vs } 191,5 \min (\mathrm{p}=0,012) \\
\text { Hospitalización: } 3,1 \text { vs } 3,8 \text { días }(\mathrm{p}=0,914) \\
\text { Pieza op: } 73,3 \text { vs } 53,8 \text { gr }(\mathrm{p}=0,029) \\
\text { Morbilidad: NE }\end{array}$ \\
\hline $\begin{array}{l}\text { Toker et al., } 2005^{17} \\
\text { Comparativo retrospectivo } \\
2001-2004 \text { (nivel } 2 b)^{*} \\
\text { Sin timoma } \\
\text { Osserman II }\end{array}$ & $\begin{array}{l}\text { VATS }+ \text { Cervicotomía selectiva }(n=32) \\
\text { EP } \quad(n=19)\end{array}$ & $\begin{array}{l}\text { Tiempo op: } 101,7 \text { vs } 82,7 \min (\mathrm{p}=0,06) \\
\text { Hosp. post op: } 2,3 \text { vs } 5,6 \text { días }(\mathrm{p}<0,001) \\
\text { Dolor: EVA } 3,1 \text { vs } 4,8 \text { puntos }(\mathrm{p}<0,001) \\
\text { Morbilidad: } 1 / 32 \text { grupo VATS }\end{array}$ \\
\hline
\end{tabular}

VATS: cirugía torácica video asistida, TE: transesternal, TAL: toracotomía anterolateral, BVATS: cirugía torácica video asistida bilateral, EP: esternotomía parcial, MGFA: clasificación clínica de la Fundación de Miastenia Gravis de América, NE: no especificado, ns: no significativo, ()*: nivel de evidencia. 
Tabla 1. Continuación

\begin{tabular}{|c|c|c|}
\hline Autor / Estudio & Abordajes (pacientes) & Resultados \\
\hline $\begin{array}{l}\text { Chang et al., } 2005^{18} \\
\text { Randomizado controlado } \\
1999-2003 \text { (nivel } 1 \text { b)* } \\
\text { Sin timoma } \\
\text { Osserman I-III }\end{array}$ & $\begin{array}{l}\text { BVATS }(\mathrm{n}=15) \\
\text { TE } \quad(\mathrm{n}=16)\end{array}$ & $\begin{array}{l}\text { Tiempo op: } 4,22 \text { vs } 1,8 \mathrm{~h}(\mathrm{p}<0,05) \\
\text { Sangrado: } 73,8 \text { vs } 155,28 \mathrm{ml}(\mathrm{p}<0,05) \\
\text { Hospitalización: } 8,22 \text { vs } 11,76(\mathrm{p}=\mathrm{ns}) \\
\text { Morbilidad: } 6 \% \text { vs } 12,5 \%(\mathrm{p}=\mathrm{ns})\end{array}$ \\
\hline $\begin{array}{l}\text { Tomulescu et al., } 2006^{19} \\
\text { Cohorte retrospectiva } \\
1999 \text { - } 2004 \text { (nivel } 2 \text { b)* } \\
\text { Sin timoma } \\
\text { MGFA IIa - V }\end{array}$ & VATS $\quad(n=106)$ & $\begin{array}{l}\text { Tiempo op: } 90 \pm 45 \text { min } \\
\text { Hospitalización: } 2,3 \text { días } \\
\text { (rango 2-6 días) } \\
\text { Morbilidad: } 9,24 \%\end{array}$ \\
\hline $\begin{array}{l}\text { Bachmann et al., } 2008^{20} \\
\text { Cohorte retrospectiva } \\
1980-2005 \text { (nivel 2b)* } \\
\text { Sin timoma } \\
\text { Osserman II-IV }\end{array}$ & $\begin{array}{ll}\text { VATS } & (\mathrm{n}=22) \\
\text { TE } & (\mathrm{n}=84)\end{array}$ & $\begin{array}{l}\text { Hospitalización: } 10,5 \text { vs } 19 \text { días }(\mathrm{p}<0,0001) \\
\text { Morbilidad: } 22,7 \% \text { vs } 19 \%(\mathrm{p}=0,765)\end{array}$ \\
\hline $\begin{array}{l}\text { Maggi et al., } 2008^{21} \\
\text { Cohorte retrospectiva } \\
1986 \text { - } 2006 \text { (nivel } 2 b)^{*} \\
\text { Con timoma }\end{array}$ & $\begin{array}{l}\text { BVATS }(\mathrm{n}=71) \\
\text { TE } \quad(\mathrm{n}=126)\end{array}$ & $\begin{array}{l}\text { Tiempo op: NE } \\
\text { Hospitalización: NE } \\
\text { Morbilidad: NE } \\
\text { Conversión: NE }\end{array}$ \\
\hline $\begin{array}{l}\text { Toker et al., } 2008^{22} \\
\text { Cohorte prospectiva } \\
2002 \text { - } 2006 \text { (nivel } 1 b)^{*} \\
\text { Sin timoma } \\
\text { Osserman NE }\end{array}$ & VATS $\quad(n=90)$ & $\begin{array}{l}\text { Tiempo op: } 64,3 \pm 27,4 \text { min } \\
\text { Hospitalización: } 2,2 \pm 1,1 \text { días } \\
\text { Dolor: EVA } 2 \pm 1,4 \text { puntos } \\
\text { Morbilidad: } 2,2 \% \\
\text { Conversión: } 2,2 \%\end{array}$ \\
\hline $\begin{array}{l}\text { Yu et al., } 2008^{23} \\
\text { Cohorte prospectiva } \\
\text { Dos años (nivel } 1 \mathrm{~b})^{*} \\
\text { Sin timoma } \\
\text { MGFA I a IIIb }\end{array}$ & $\begin{array}{l}\text { VATS }+ \text { cervicotomía } \\
(\mathrm{n}=36)\end{array}$ & $\begin{array}{l}\text { Tiempo op: } 162 \mathrm{~min} \\
\text { (rango } 132-210 \mathrm{~min} \text { ) } \\
\text { Pieza op: timo } 44,2 \mathrm{gr} \text {; } \\
\text { grasa mediastínica } 32,5 \mathrm{gr} \text {; } \\
\text { grasa cervical } 3,6 \mathrm{gr} \\
\text { Morbilidad: } 8,33 \%\end{array}$ \\
\hline $\begin{array}{l}\text { Pompeo et al., } 2009^{25} \\
\text { Cohorte retrospectiva } \\
1995 \text { - } 2003 \text { (nivel } 2 b)^{*} \\
\text { Sin timoma } \\
\text { MGFA I-IV }\end{array}$ & $\begin{array}{l}\text { VATS }+ \text { cervicotomía } \\
(\mathrm{n}=32)\end{array}$ & $\begin{array}{l}\text { Tiempo op: } 150 \text { min } \\
\text { (rango 140-180) } \\
\text { Hospitalización: } 4 \text { días } \\
\text { (rango 3-5,5) } \\
\text { Conversión: } 6 \%\end{array}$ \\
\hline $\begin{array}{l}\text { Meyer et al., } 2009^{26} \\
\text { Cohorte retrospectiva } \\
1992 \text { - } 2006 \text { (nivel 2b)* } \\
\text { Timoma NE } \\
\text { MGFA I-V }\end{array}$ & $\begin{array}{ll}\text { VATS } & (\mathrm{n}=48) \\
\text { TE } & (\mathrm{n}=47)\end{array}$ & $\begin{array}{l}\text { Tiempo op: } 128 \text { vs } 119 \min (p=0,22) \\
\text { Hospitalización: } 1,9 \text { vs } 4,6 \text { días }(p<0,001) \\
\text { Morbilidad: } 4,2 \% \text { vs } 16,2 \%(p=0,007)\end{array}$ \\
\hline $\begin{array}{l}\text { Lin et al., } 2010^{27} \\
\text { Cohorte retrospectiva } \\
1995-2004 \text { (nivel } 2 b)^{*} \\
\text { Sin timoma } \\
\text { MGFA I-V }\end{array}$ & $\begin{array}{ll}\text { VATS } & (\mathrm{n}=38) \\
\text { TE } & (\mathrm{n}=22)\end{array}$ & $\begin{array}{l}\text { Tiempo op: } 169,2 \text { vs } 177 \mathrm{~min}(\mathrm{p}=0,57) \\
\text { Sangrado: } 125,7 \text { vs } 186,8 \mathrm{ml}(\mathrm{p}=0,024) \\
\text { Hospitalización: } 5,6 \text { vs } 8,1 \text { días }(\mathrm{p}=0,008) \\
\text { Morbilidad: } 5 \% \text { vs } 5 \%\end{array}$ \\
\hline $\begin{array}{l}\text { Huang et al., } 2011^{28} \\
\text { Comparativo prospectivo } \\
\text { pareado } \\
1998 \text { - } 2003 \text { (nivel } 1 \mathrm{~b})^{*} \\
\text { Con y sin timoma } \\
\text { Osserman I-III }\end{array}$ & $\begin{array}{ll}\text { VATS } & (\mathrm{n}=33) \\
\text { TE } & (\mathrm{n}=66)\end{array}$ & $\begin{array}{l}\text { Tiempo op: } 207,3 \text { vs } 172,8 \min (p=0,003) \\
\text { Sangrado: } 88,5 \text { vs } 226,8 \mathrm{ml}(\mathrm{p}<0,001) \\
\text { Pieza op: } 41,1 \text { vs } 51 \text { gr }(\mathrm{p}=0,133) \\
\text { Hospitalización: } 7,5 \text { vs } 8,3 \text { días }(\mathrm{p}=0,225) \\
\text { Morbilidad: } 24,2 \% \text { vs } 15,1 \%(\mathrm{p}=0,269)\end{array}$ \\
\hline $\begin{array}{l}\text { Lee et al., } 2011^{29} \\
\text { Comparativo retrospectivo } \\
2006 \text { - } 2009 \text { (nivel } 2 b)^{*} \\
\text { Con y sin timoma } \\
\text { MGFA I-IV }\end{array}$ & $\begin{array}{ll}\text { BVATS } & (\mathrm{n}=55) \\
\text { TE } & (\mathrm{n}=59)\end{array}$ & $\begin{array}{l}\text { Tiempo op: } 112,2 \text { vs } 130,7 \mathrm{~min}(\mathrm{p}=0,908) \\
\text { Sangrado: } 34 \text { vs } 122,6 \mathrm{ml}(\mathrm{p}<0,001) \\
\text { Pieza op: } 72,5 \text { vs } 74,1 \mathrm{gr}(\mathrm{p}=0,63) \\
\text { Hospitalización: } 6,28 \text { vs } 14,6 \text { días }(\mathrm{p}=0,01) \\
\text { Morbilidad: } 2 \% \text { vs } 5 \%(\mathrm{p}=0,34)\end{array}$ \\
\hline
\end{tabular}


Tabla 2. Resultados clínicos: remisión completa estable (RCE), mejoría clínica y tiempo de seguimiento

\begin{tabular}{|c|c|c|c|}
\hline Autor / Año / Abordaje & RCE & Mejoría clínica & Seguimiento \\
\hline $\begin{array}{l}\text { Yim et al., } 1995^{10} \\
\text { VATS }\end{array}$ & $\mathrm{NE}$ & $100 \%$ & $\begin{array}{l}\text { Media: } 10 \text { meses } \\
\text { (rango } 2 \text { - } 20 \text { meses) }\end{array}$ \\
\hline $\begin{array}{l}\text { Mineo et al., } 2000^{11} \\
\text { VATS }\end{array}$ & $36 \%$ & $96 \%$ & Media: $39,6 \pm 15$ meses \\
\hline $\begin{array}{l}\text { Savcenko et al., } 2002^{12} \\
\text { VATS }\end{array}$ & $14 \%$ & $83 \%$ & Media: $53,24 \pm 32,57$ meses \\
\hline $\begin{array}{l}\text { Wright et al., } 2002^{13} \\
\text { VATS }\end{array}$ & $27 \%$ & $81 \%$ & Media: $19,53 \pm 12,74$ meses \\
\hline $\begin{array}{l}\text { Ruckert el al., } 2003^{14} \\
\text { VATS vs TE vs TAL }\end{array}$ & $\begin{array}{l}28 \% \text { vs } 22 \% \text { vs } 31 \% \\
(p=n s)\end{array}$ & $\mathrm{NE}$ & Media: 24 meses \\
\hline $\begin{array}{l}\text { Mantegazza et al., } 2003^{15} \\
\text { BVATS vs TE }\end{array}$ & $\begin{array}{l}50,6 \% \text { vs } 48,7 \% \\
(p=N E)\end{array}$ & $\mathrm{NE}$ & Media: 46,8 meses \\
\hline $\begin{array}{l}\text { Hsu et al., } 2004^{16} \\
\text { BVATS vs VATS }\end{array}$ & $\begin{array}{l}33,3 \% \text { vs } 40 \% \\
(p=0,722)\end{array}$ & $\mathrm{NE}$ & Media: 18,5 meses (rango 6 - 30 meses) \\
\hline $\begin{array}{l}\text { Toker et al., } 2005^{17} \\
\text { VATS }\end{array}$ & $\mathrm{NE}$ & $\mathrm{NE}$ & $\mathrm{NE}$ \\
\hline $\begin{array}{l}\text { Chang et al., } 2005^{18} \\
\text { BVATS vs TE }\end{array}$ & $\begin{array}{l}33 \% \text { vs } 37 \% \\
(p=0,667)\end{array}$ & $\begin{array}{l}80 \% \text { vs } 93,75 \% \\
(p=N E)\end{array}$ & $\begin{array}{l}\text { Media: } 33 \pm 18,72 \text { vs } 29,38 \pm 11,84 \\
(p=n s)\end{array}$ \\
\hline $\begin{array}{l}\text { Tomulescu et al., } 2006^{19} \\
\text { VATS }\end{array}$ & $39,25 \%$ & $97,19 \%$ & Media: 36,4 meses (rango $12-74$ meses) \\
\hline $\begin{array}{l}\text { Bachmann et al., } 2008^{20} \\
\text { VATS vs TE }\end{array}$ & $\begin{array}{l}47,6 \% \text { vs } 35,1 \% \\
(p=0,319)\end{array}$ & $\begin{array}{l}100 \% \text { vs } 77,9 \% \\
(p=0,019)\end{array}$ & $\begin{array}{l}\text { Mediana: } 8 \text { años } \\
\text { (rango } 1-25 \text { años) }\end{array}$ \\
\hline $\begin{array}{l}\text { Maggi et al., } 2008^{21} \\
\text { BVATS vs TE }\end{array}$ & $\begin{array}{l}11,3 \% \text { vs } 8,7 \% \\
(p=0,1090)\end{array}$ & $\mathrm{NE}$ & 20 años \\
\hline $\begin{array}{l}\text { Toker et al., } 2008^{22} \\
\text { VATS }\end{array}$ & $45 \%$ & $\mathrm{NE}$ & Rango: 39,6 - 64,8 meses \\
\hline $\begin{array}{l}\text { Yu et al., } 2008^{23} \\
\text { VATS + cervicotomía }\end{array}$ & $27,8 \%$ & $88,9 \%$ & Media: 24 meses \\
\hline $\begin{array}{l}\text { Shiono et al., } 2008^{24} \\
\text { BVATS vs TE }\end{array}$ & $\begin{array}{l}42 \% \text { vs } 40 \% \\
(p=n s)\end{array}$ & $\begin{array}{l}85 \% \text { vs } 84 \% \\
(p=n s)\end{array}$ & Mediana: 38,8 meses (rango 3-68) \\
\hline $\begin{array}{l}\text { Pompeo et al., } 2009^{25} \\
\text { VATS + cervicotomía }\end{array}$ & $44 \%$ & $84 \%$ & Mediana: 119 meses (rango 60-156) \\
\hline $\begin{array}{l}\text { Meyer et al., } 2009^{26} \\
\text { VATS vs TE }\end{array}$ & $\begin{array}{l}34,9 \% \text { vs } 15,8 \% \\
(p=n s)\end{array}$ & $\begin{array}{l}95 \% \text { vs } 97,4 \% \\
(p=n s)\end{array}$ & Media: $6,4 \pm 4$ vs $4,3 \pm 2,9$ años \\
\hline $\begin{array}{l}\text { Lin et al., } 2010^{27} \\
\text { VATS vs TE }\end{array}$ & $32 \%$ vs $36 \%$ & $\mathrm{NE}$ & Media: 38,5 meses \\
\hline $\begin{array}{l}\text { Huang et al., } 2011^{28} \\
\text { VATS vs TE }\end{array}$ & $\begin{array}{l}42,4 \% \text { vs } 60,6 \% \\
(p=0,087)\end{array}$ & $\begin{array}{l}87,9 \% \text { vs } 90,1 \% \\
(p=0,637)\end{array}$ & Media: $77,2 \pm 19,5$ vs $117,7 \pm 53,3$ meses \\
\hline Lee et al., $2011^{29}$ & $\mathrm{NE}$ & $\mathrm{NE}$ & $\mathrm{NE}$ \\
\hline
\end{tabular}

VATS: cirugía torácica video asistida, TE: transesternal, TAL: toracotomía anterolateral, BVATS: cirugía torácica video asistida bilateral, NE: no especificado, ns: no significativo.

con la timectomía Transesternal (TE) y describen que la primera presentaba menor tiempo operatorio, de hospitalización y menor requerimiento de analgésicos opioides $(\mathrm{p}<0,05)$. Mineo et al, $(2000)^{11}$ encontraron que los resultados de la timectomía por VATS eran comparables con las técnicas invasivas, con menor morbilidad y mejor resultado estético. Además los resultados muestran que mientras más precoz es la indicación quirúrgica, mejores son los resultados clínicos, independiente de la gravedad de los síntomas preoperatorios. El análisis univariado mostró que la duración de la enfermedad $<12$ meses 
se correlacionó significativamente con la mejoría a 24 meses $(p=0,036)$. Savcenko et al, $(2002)^{12}$ y Wright et al, $(2002)^{13}$ encontraron que la timectomía por VATS presentaba menor tiempo de hospitalización y baja morbilidad. Ruckert el al, $(2003)^{14}$ compararon la timectomía por VATS, TE y por toracotomía anterolateral (TAL) y encontraron que la VATS era equivalente a la TE y TAL en términos de remisión $(\mathrm{p}>0,05)$, con mayor tiempo operatorio $(\mathrm{p}<0,001)$ pero menor morbilidad $(\mathrm{p}<0,05)$ y mayor satisfacción estética. Mantegazza et al, $(2003)^{15}$ compararon la timectomía por VATS bilateral (BVATS) asociada a cervicotomía y encontraron resultados terapéuticos similares a la cirugía por vía TE clásica y mejores que los descritos en otras series por VATS unilaterales, con baja morbilidad y resultados estéticos superiores. Además tanto el análisis univariado como multivariado mostraron que la hiperplasia tímica y la ausencia de inmunosupresión preoperatoria se correlacionaban significativamente con la probabilidad de obtener una remisión completa estable (RCE). Hsu et al (2004) $)^{16}$, compararon la timectomía por BVATS con asistencia subxifoidea con la VATS. La BVATS tuvo menor tiempo operatorio $(\mathrm{p}=0,012)$, ofreciendo mejor visión, mayor cantidad de tejido resecado $(p=0,029)$, sin diferencias en los resultados clínicos a corto plazo $(p=0,722)$. Toker et al $(2005)^{17}$, compararon la timectomía por VATS asociada a mini cervicotomía selectiva con la timectomía por esternotomía parcial (EP) y encontraron que la VATS era segura, con mayor tiempo operatorio, no significativo, menor dolor postoperatorio $(p<0,001)$ y menos días de hospitalización $(\mathrm{p}<0,001)$. Chang et al $(2005)^{18}$, compararon la timectomía por BVATS con la timectomía TE. La BVATS, requirió mayor tiempo operatorio $(p<0,05)$, con menor pérdida sanguínea $(\mathrm{p}<0,05)$, mejor resultado estético y resultados a largo plazo equivalentes. Tomulescu et al (2006) ${ }^{19}$, analizaron una serie de pacientes abordados por VATS y encontraron resultados peri operatorios mejores a los descritos para los abordajes invasivos. Además la edad menor de 40 años al diagnóstico y la cirugía indicada en forma precoz (no detallado el tiempo) fueron predictores de remisión completa estable (RCE) en forma significativa $(p=0,04$ y $\mathrm{p}=0,03$, respectivamente). No se observó diferencias entre los abordajes derecho e izquierdo en cuanto a evolución postoperatoria ni remisión. Bachmann et al (2008) ${ }^{20}$, compararon la timectomía por VATS con la timectomía TE. La VATS tuvo menor hospitalización $(p<0,0001)$ y mayor tasa de RCE a largo plazo, no significativa $(p=0,319)$. Un Osserman IV preoperatorio se asoció a mayor tasa de complicaciones $(p=0,041)$, la edad mayor de 45 años y la presencia de timoma fueron predictores de supervivencia. Maggi et al $(2008)^{21}$, revisaron los resultados de timectomía por BVATS y TE en pacientes con MG asociada a timoma tratando de identificar variables que influenciaran la historia natural de la enfermedad. La BVATS resultó ser efectiva para la MG con timoma y no aumentó la tasa de recurrencia $(p=0,1523)$. Ningún paciente con timoma de alto grado (etapa IV o tipo B) alcanzó la RCE. La MG asociada a timoma tuvo peor pronóstico que la sin timoma. Toker et al $(2008)^{22}$, en una serie de pacientes abordados por VATS describen que es una técnica segura, con mejor resultado estético, con baja morbilidad y resultados comparables a las técnicas invasivas. Yu et al (2008) ${ }^{23}$, en una serie de pacientes operados por VATS asociada a cervicotomía señalan que es una técnica segura y efectiva, y que el abordaje cervical permitiría la resección de tejido tímico residual (sin determinar el verdadero impacto en los resultados clínicos). Shiono et al $(2008)^{24}$, compararon la timectomía por BVATS asociada a cervicotomía con la TE, la BVATS asociada a cervicotomía tuvo mayor tiempo operatorio $(\mathrm{p}<0,001)$, menor sangrado $(\mathrm{p}<0,001)$ y similar cantidad de tejido resecado $(\mathrm{p}=0,369)$. Pompeo et al $(2009)^{25}$, en una serie de pacientes abordados por VATS asociada a cervicotomía concluyen que la duración de síntomas $<12$ meses y la ausencia de compromiso orofaríngeo eran predictores de buenas respuestas $(p=0,02)$, y que la presencia de anticuerpos anti-MuSK se asociaron a una peor respuesta $(\mathrm{p}=0,0007)$. Meyer et al $(2009)^{26}$, compararon la timectomía por VATS con la TE, encontrando que la VATS era segura, con menor morbilidad $(\mathrm{p}=$ $0,007)$ y días de hospitalización $(\mathrm{p}<0,001)$. Lin et al $(2010)^{27}$, compararon la timectomía por VATS con la TE, señalando que la VATS es una vía tan segura y eficaz como la TE. Con menor tiempo de hospitalización $(p=0,008)$. Se identificaron como posibles factores predictores de alcanzar RCE la edad menor de 40 años $(p=0,002)$, el hipertiroidismo $(p=0,003)$ y la hiperplasia tímica $(p=0,041)$. Huang et al (2011) ${ }^{28}$, compararon los resultados de la timectomía por VATS con la TE. La VATS tuvo mayor tiempo operatorio $(\mathrm{p}=0,003)$, menor sangrado $(p<0,001)$, sin diferencias significativas en la cantidad de tejido resecado, tiempo de hospitalización ni morbilidad, pero la RCE fue mayor en la vía TE. Por último, Lee et al (2011) $)^{29}$, compararon la timectomía por BVATS con la TE. La BVATS tuvo menor sangrado intraoperatorio $(p<0,001)$ y tiempo de hospitalización $(\mathrm{p}=0,01)$, sin diferencias en el tiempo operatorio $(p=0,908)$ y la cantidad de tejido resecado $(\mathrm{p}=0,63)$. Si bien la morbilidad fue similar, no se consideraron las crisis miasténicas que fueron $0 \%$ versus $10,2 \%(\mathrm{p}=0,02)$ a favor del grupos BVATS. 


\section{Discusión}

El tratamiento de la MG involucra el tratamiento médico y el quirúrgico. Desde 1939, la timectomía se ha transformado en parte importante del tratamiento de esta enfermedad y para muchos debería ser el pilar terapéutico de los pacientes con $\mathrm{MG}$ generalizada. La timectomía en pacientes con MG ocular es todavía controversial, porque no solamente pareciera responder peor a la timectomía, sino que además, tiene un mejor pronóstico comparada con MG generalizada. Por otro lado, se ha documentado que entre 30 a $70 \%$ de los pacientes con síntomas puramente oculares desarrollarán eventualmente la forma generalizada, lo que apoyaría la cirugía ${ }^{30}$.

El objetivo técnico de la timectomía es la resección completa de todo el tejido tímico y el objetivo terapéutico la remisión de la enfermedad. Está establecido que el timo toma un rol central en la patogénesis de esta enfermedad autoinmune y que las resecciones incompletas han sido causa de falla terapéutica y de reoperaciones ${ }^{31-35}$. Dado que el timo no es una estructura única, sino que se distribuye de forma discontinua en la grasa mediastínica desde el diafragma hasta el cuello, la resección debe incluir todo este tejido (timectomía extendida). En la actualidad no existe un examen que permita predecir la presencia y localización de tejido tímico microscópico en el intraoperatorio o informar si ha quedado tejido residual en el postoperatorio. La única forma de medir si la resección tímica fue completa se basa en la tasa de remisión más alta.

Cuál es el mejor abordaje para realizar la timectomía sigue siendo tema de discusión. El abordaje óptimo debería ser capaz de combinar la mínima invasividad y la máxima radicabilidad. La timectomía transcervical-transesternal "máxima" ha sido descrita como la técnica contra la cual los otros procedimientos deben ser medidos ${ }^{36}$.

Las técnicas mínimamente invasivas son variadas, e incluyen la VATS unilateral derecha o izquierda, la VATS bilateral, asociado o no con cervicotomía o exposición subxifoidea.

Jaretzki en $1997^{37}$, analizó las controversias en relación a las técnicas descritas y sus resultados. Describió los distintos abordajes estimando en forma arbitraria los porcentajes de tejido tímico resecado: timectomía transcervical-transesternal máxima (98$100 \%)$, transesternal extendida (85-95\%), VATS (80-85\%), transcervical extendida (75-80\%), transesternal clásica $(70-75 \%)$ y transcervical básica (40-50\%), concluyendo que la evidencia indicaba una relación directa entre las extensión del tejido tímico y los resultados en cuanto a remisión y tiempo de seguimiento, así la timectomía máxima constituiría la mejor opción terapéutica.
Zahid et $\mathrm{al}^{38}$, en una revisión para determinar cuál era la mejor vía para la timectomía por MG, analizaron 15 comunicaciones, concluyendo que si bien no existía consenso, la VATS al parecer sería equivalente a la vía transesternal en cuanto a mortalidad y RCE, con menor tiempo de hospitalización, menor pérdida sanguínea y mayor satisfacción estética del paciente.

Decidir la mejor vía de abordaje para la timectomía, se torna difícil cuando no se cuenta con estudios de metodología adecuada, es decir, ensayos clínicos aleatorios doble ciego, que comparen las distintas técnicas quirúrgicas. Además, la heterogeneidad de los pacientes, de las técnicas quirúrgicas utilizadas, lo fluctuante de la enfermedad, el uso de distintas clasificaciones para evaluar la gravedad de la enfermedad y los resultados postoperatorios, así como el escaso número de pacientes que incluyen los estudios, complican aún más los análisis. Por otra parte, la definición de parámetros como la remisión o mejoría, no siempre es la misma, y hace las comparaciones un tanto subjetivas.

En esta revisión seleccionamos 20 artículos para analizar. Trece estudios retrospectivos y 7 prospectivos. Ocho artículos correspondieron a series de casos y 12 fueron estudios comparativos entre $2 \mathrm{o}$ más abordajes quirúrgicos. El diseño de los estudios difiere en su desarrollo, objetivos evaluados y períodos de seguimiento. Sin embargo, los resultados muestran que las técnicas mínimamente invasivas (video-asistida) serían una vía segura, con tasas de morbilidad bajas, menor tiempo de hospitalización, mejor resultado estético y tasas de remisión completa o de mejoría al parecer equivalentes a las vías de abordaje clásicas. Si bien el tiempo operatorio es mayor, existe una tendencia a disminuir a medida que el número de intervenciones aumenta, como lo mostraron Mantegazza et a $\mathrm{l}^{15}$.

Para concluir, podemos decir que la cirugía mínimamente invasiva (video-asistida) para la timectomía en pacientes con MG es una vía segura, con tasas de morbilidad bajas, menor tiempo de hospitalización, buen resultado estético y tasas de remisión completa o de mejoría al parecer equivalentes a las vías de abordaje clásicas; pero estos resultados deben evaluarse con cautela ya que no existe suficiente evidencia para asegurar que la cirugía mínimamente invasiva (video-asistida) es el mejor abordaje.

\section{Referencias}

1. Drachman DB. Myasthenia Gravis. N Engl J Med. 1994;330:1797-810.

2. Suárez GA. Myasthenia gravis: diagnosis and treatment. Rev Neurol. 1999;29:162-5. 
3. Osserman KE, Genkins G. Studies In Myasthenia Gravis: Review Of A 20-Year Experience In Over 1,200 Patients. J Mount Sinai Hosp. 1971;38:497-537.

4. Drachman DB, Adams RN, Josifek LF, Self SG. Functional activities of autoantibodies to acetylcoline receptors and the clinical severity of myasthenia gravis. New Engl J Med. 1982;307:769-75.

5. Jaretzki A, Barohn R, Ernstoff RM, Kaminski HJ, Keesey JC, Penn AS, et al. Myasthenia gravis: recommendations for clinical research standards. Task Force of the Medical Scientific Advisory Board of the Myasthenia Gravis Foundation of America. Ann Thorac Surg. 2000;70:327-34.

6. Schumacher R, Roth J. Thymektomie Bei Cenem Fall Von Morbus Basedowii Mit Myasthenie. Med Chir. 1912;25:746

7. Blalock A, Mason MF, Morgan HJ, Riven SS. Myasthenia Gravis And Tumors Of The Thymic Region: Report Of A Case In Which The Tumor Was Removed. Ann Surg. 1939;110:544-61.

8. Blalock A, Mcgehee Ha, Ford Fr. The Treatment Of Myasthenia Gravis By Removal Of The Thymus Gland. JAMA 1941;117:1529.

9. Buckingham J, Howard F Jr, Bernantz P, Payne WS, Harrison EG Jr, O'Brien PC et al. The Value Of Thymectomy In Myasthenia Gravis: A Computer-Assisted Matched Study. Ann Surg. 1976;184:453-8.

10. Yim AP, Kay RL, Ho JK. Video-assisted thoracoscopic thymectomy for myasthenia gravis. Chest 1995;108:1440-3.

11. Mineo TC, Pompeo E, Lerut, TE, Bernardi G, Coosemans W, Nofroni I. Thoracoscopic thimectomy in autoimmune Myasthenia: results of left-side approach. Ann Thorac Surg. 2000;69:537-41.

12. Savcenko M, Wendt G, Prince S, Mack M. Videoassisted thymectomy for myasthenia gravis: an update of a single institution experience. Eur J Cardiothorac Surg. 2002;22:978-83.

13. Wright G, Barnett S, Clarke CP. Video-assisted thoracoscopic thymectomy for myasthenia gravis. Intern Med J. 2002;32:367-71.

14. Rückert JC, Sobel HK, Göhring S, Einhäupl KM, Müller JM. Matched-pair comparison of three different approaches for thymectomy in myasthenia gravis. Surg Endosc. 2003;17:711-5.

15. Mantegazza R, Baggi F, Bernasconi P, Antozzi C, Confalonieri P, Novellino L et al. Video-assisted thoracoscopic extended thimectomy and extended trasnsesternal thymectomy (T-3b) in non-thymomatous myasthenia gravis patients: remission after 6 years of follow-up. J Neurol Sci. 2003;212:31-6.

16. Hsu CP, Chuang CY, Hsu NY, Chen CY. Comparison between the right side and subxiphoid bilateral approaches in performing video-assisted thoracoscopic extended thymectomy for myasthenia gravis. Surg Endosc. 2004;18:821-4
17. Toker A, Eroglu O, Zidaye S, Tanju S, Senturk M, Dilege $\mathrm{S}$ et al. Comparison of early postoperative results of thymectomy: partial esternotomy vs videothoracoscopy. Thorac Cardiov Surg. 2005;53:110-3.

18. Chang PC, Chou SH, Kao EL, Cheng YJ, Chuang HY, Liu CK, et al. Bilateral video-assisted thoracoscopic thymectomy vs. extended transsternal thymectomy in myasthenia gravis: a prospective study. Eur Surg Res. 2005;4:199-203.

19. Tomelescu V, Ion V, Kosa A, Sgarbura O, Popescu I. Thoracoscopic thymectomy mild-term results. Ann Thorac Surg. 2006;82:1003-7.

20. Bachmann K, Burkhardt D, Schreiter I, Kaifi J, Busch $\mathrm{C}$, Thayssen G, et al. Long-term outcome and quality of life after open and thoracoscopic thymectomy for myasthenia gravis: analysis of 131 patients. Surg Endosc. 2008;22:2470-7.

21. Maggi L, Andreetta F, Antozzi C, Baggi F, Bernasconi $\mathrm{P}$, Cavalcante $\mathrm{P}$, et al. Thymoma-associated myasthenia gravis: outcome, clinical and pathological correlations in 197 patients on a 20-year experience. J Neuroimmunol. 2008;201-202:237-44.

22. Toker A, Tanju S, Sungur Z, Parman Y, Senturk M, Serdaroglu P, et al. Videothoracoscopic thymectomy for nonthymomatous myasthenia gravis: results of 90 patients. Surg Endosc. 2008;22:912-6.

23. Yu L, Shan M, Jiang J, Jing Y, Zang N, Wang T, et al. Combined transcervical and unilateral-thoracoscopic Thymectomy for Myasthenia Gravis: 2 years of followup. Surg Laparosc Endosc Percutan Tech. 2008;18:48992.

24. Shiono H, Kadota Y, Hayashi A, Okumura M. Comparision of outcomes after extends thymectomy for myasthenia gravis: bilateral thoracoscopic approach versus sternotomy. Surg Laparosc Endosc Percutan Tech. 2009;19:424-7.

25. Pompeo E, Tacconi F, Massa R, Mineo D, Nahmias $\mathrm{S}$, Mineo TC. Long-term outcome of thoracoscopic extended thymectomy for nonthymomatous myasthenia gravis. Eur J Cardiothoracic Surg. 2009;36:164-9.

26. Meyer DM, Herbert MA, Sobhani NC, Tavakolian P, Duncan A, Bruns M, et al. Comparative clinical outcomes of thymectomy for myasthenia gravis performed by extended transsternal and minimally invasive approaches. Ann Thorac Surg. 2009;87:385-90.

27. Lin MW, Chang YL, Huang PM, Lee YC. Thymectomy for non-thymomatous myasthenia gravis: a comparison of surgical methods and analysis of prognostic factors. Eur J Cardiothorac Surg. 2010;37:7-12.

28. Huang CS, Cheng CY, Hsu HS, Kao KP, Hsieh CC, Hsu $\mathrm{WH}$, et al. Video-assisted thoracoscopic surgery versus sternotomy in the treating myasthenia gravis: comparision by a case matched study. Surg Today 2011;41:33845 .

29. Lee CY, Kim DJ, Lee JG, Park IK, Bae MK, Chung KY. Bilateral video assisted thoracoscopic thymectomy 
has a surgical extent similar to that of transesternal extended thimectomy with more favorable early surgical outcomes for myasthenia gravis patients. Surg Endosc. 2011;25:849-54.

30. Gronseth GS, Barohn RJ. Practice parameter: thimectomy for autoimmune myasthenia gravis (an evidence-base review): report of the Quality Standars Subcommitte of the American Academy Of Neurology. Neurology 2000;55:7-15.

31. Henze A, Biberfeld P, Christensson B, Matell G, Pirskanen R. Failing transcervical thymectomy in myasthenia gravis, an evaluation of transsternal re-exploration. Scand J Thorac Cardiovasc Surg. 1984:18:235-8.

32. Jaretzki A 3rd, Penn AS, Younger DS, Wolff M, Olarte MR, Lovelace RE, et al. "Maximal" thymectomy for myasthenia gravis: results. J Thorac Cardiovasc Surg. 1988;95:747-57.

33. Masaoka A, Monden Y, Seike Y, Tanioka T, Kagotani K. Reoperation after transcervical thymectomy for myasthenia gravis. Neurology 1982;32:83-5.

34. Miller RG, Filler-Katz A, Kiprov D, Roan R. Repeat thymectomy in chronic refractory myasthenia gravis. Neurology 1991;41:923-4.

35. Rosenberg M, Jauregui WO, De Vega ME, Herrera MR, Roncoroni AJ. Recurrence of thymic hyperplasia after thymectomy in myasthenia gravis: its importance as a cause of failure of surgical treatment. Am J Med. 1983;74:78-82.

36. Cooper J, Jaretzki A 3rd, Mulder DG, Papatestas AS. Symposium: thymectomy for myasthenia gravis. Contemp Surg. 1989;34:65-86.

37. Jaretzki A 3rd. Thimectomy for myasthenia gravis: Analysis of the controversies regarding technique and results. Neurology 1997;48(Suppl 5):S52-S63.

38. Zahid I, Sharif S, Routledge T, Scarci M. Video-assisted thoracoscopic. Surgery or transsternal thymectomy in the treatment of myasthenia gravis? Interact Cardiovasc Thorac Surg. 2011;12:40-6. 